$03,09,13$

\title{
Диэлектрические и оптические свойства кубических монокристаллов SiC, GeC и SnC: модельные оценки
}

\author{
(ㄷ. С.Ю. Давыдов, А.А. Лебедев
}

Физико-технический институт им. А.Ф. Иофрфе РАН,

Санкт-Петербург, Россия

Email: Sergei_Davydov@mail.ru

Поступила в Редакцию 25 августа 2021 г.

В окончательной редакции 25 августа 2021 г.

Принята к публикации 26 сентября 2021 г.

В рамках модели связывающих орбиталей Харрисона для кубических карбидов элементов IV группы получены аналитические выражения для высоко- и низкочастотных диэлектрических восприимчивостей и проницаемостей, линейного электрооптического коэффициента, фотоупругих постоянных и зависимостей диэлектрических проницаемостей от давления.

Ключевые слова: диэлектрические восприимчивости и проницаемости, линейный электрооптический коэффициент, фотоупругие постоянные.

DOI: 10.21883/FTT.2022.01.51833.193

\section{1. Введение}

Интерес к свойствам монокристаллов карбида кремния, давно и интенсивно изучаемого [1], по-прежнему велик. Достаточно сказать, что каждые два года проводятся международная и европейская конференции по карбиду кремния и родственным материалам (ICSCRM и ECSCRM). В последнее время возросло внимание к кубическому политипу $3 \mathrm{C}-\mathrm{SiC}$, который обладает максимальной среди политипов $\mathrm{SiC}$ подвижностью электронов $1200 \mathrm{~cm}^{2} / \mathrm{V} \cdot \mathrm{s}$ [2], не зависящей от кристаллографического направления.

Вопрос о возможности существования монокристаллов $\mathrm{GeC}$ и $\mathrm{SnC}$ и их свойствах возник сравнительно недавно [3-8]. В соответствующих теоретических исследованиях (расчетах из первых принципов) основное внимание уделялось стабильности тех или иных кристаллических структур, зонному спектру и упругости. В настоящей работе мы с единой точки зрения рассмотрим диэлектрические и оптические свойства кубических карбидов $X \mathrm{C}$, где $X=\mathrm{Si}, \mathrm{Ge}, \mathrm{Sn}$. При этом мы воспользуемся моделью связывающих орбиталей Харрисона [9-11], хорошо зарекомендовавшей себя при описании тетраэдрических полупроводников.

\section{2. Диэлектрические свойства карбидов}

Определив линейную $\chi^{(1)}$ и квадратичную $\chi^{(2)}$ диэлектрические восприимчивости как коэффициенты разложения поляризации кристалла $\mathbf{P}$ по напряженности электрического поля $\mathbf{E}$, т. е. в виде

$$
P_{i}=\sum_{j} \chi_{i j}^{(1)} E_{j}+\sum_{j k} \chi_{i j k}^{(2)} E_{j} E_{k}+\ldots
$$

можно показать [12,13], что вклады электронной подсистемы в эти характеристики равны

$$
\chi_{1}^{\mathrm{el}}=\frac{n_{\mathrm{e}}(e \gamma d)^{2} \alpha_{\mathrm{c}}^{3}}{12 V_{2}}, \quad \chi_{14}^{\mathrm{el}}=\frac{\sqrt{3} n_{\mathrm{e}}(e \gamma d)^{3} \alpha_{\mathrm{c}}^{4} \alpha_{\mathrm{p}}}{48 V_{2}^{2}},
$$

тогда как ионные (решеточные) вклады имеют вид

$$
\begin{gathered}
\chi_{1}^{\text {ion }}=\frac{n_{\mathrm{e}}(e \gamma d)^{2} \alpha_{\mathrm{p}}^{2}\left(1+2 \alpha_{\mathrm{c}}^{2}\right)}{24 \alpha_{\mathrm{c}} V_{2}}, \\
\chi_{14}^{\text {ion }}=\frac{\sqrt{3} n_{\mathrm{e}}(e \gamma d)^{3} \alpha_{\mathrm{c}}^{2} \alpha_{\mathrm{p}}\left(1-2 \alpha_{\mathrm{p}}^{2}\right)}{48 V_{2}^{2}} .
\end{gathered}
$$

Для суммарных (низкочастотных) значений линейных и квадратичных восприимчивостей получаем следующие выражения:

$$
\begin{gathered}
\chi_{1}=\chi_{1}^{\mathrm{el}}(1+\vartheta), \quad \vartheta=\frac{\alpha_{\mathrm{p}}^{2}\left(1+2 \alpha_{\mathrm{c}}^{2}\right)}{2 \alpha_{\mathrm{c}}^{4}}, \\
\chi_{14}=\frac{\sqrt{3} n_{\mathrm{e}}(e \gamma d)^{3} \alpha_{\mathrm{c}}^{2} \alpha_{\mathrm{p}}}{48 V_{2}^{2}} .
\end{gathered}
$$

Здесь $V_{2}=3.22\left(\hbar^{2} / m d^{2}\right)-$ ковалентная энергия $\sigma$-связи $\mathrm{sp}^{3}$-орбиталей атомов $A$ и $B$, где $\hbar-$ приведенная постоянная Планка, $m$ - масса свободного электрона, $d=a \sqrt{3} / 4-$ расстояние между ближайшими соседями в структуре сфалерита с постоянной решетки $a$. В отличие от $[12,13]$, мы полагаем $V_{2}>0$; $\alpha_{\mathrm{c}}=V_{2} / \sqrt{V_{2}^{2}+V_{3}^{2}}$ и $\alpha_{\mathrm{p}}=\sqrt{1-\alpha_{\mathrm{c}}^{2}}-$ ковалентность и полярность связи соответственно; $V_{3}=\left|\varepsilon_{\mathrm{h}}^{A}-\varepsilon_{\mathrm{h}}^{B}\right| / 2-$ полярная энергия связи, где $\varepsilon_{\mathrm{h}}^{A(B)}=\left(\varepsilon_{\mathrm{s}}^{A(B)}+3 \varepsilon_{\mathrm{p}}^{A(B)}\right) / 4-$ энергии $\mathrm{sp}^{3}$-орбиталей и $\varepsilon_{\mathrm{s}(\mathrm{p})}^{A(B)}-$ энергия $\mathrm{s}(\mathrm{p})$-состояния атома $A(B) ; n_{\mathrm{e}}=32 / a^{3}-$ плотность электронов, $e-$ 
Таблица 1. Исходные параметры: расстояние между ближайшими соседями $d$, ковалентная $V_{2}$ и полярная $V_{3}$ энергии, ковалентность $\alpha_{\mathrm{c}}$ и полярность $\alpha_{\mathrm{p}}$ связи $X-C$. Верхний ряд значений - расчет по таблицам Манна [11], нижний ряд расчет по таблицам Хермана-Скиллмана [9]

\begin{tabular}{c|c|c|c}
\hline Кристалл & $\mathrm{SiC}$ & $\mathrm{GeC}$ & $\mathrm{SnC}$ \\
\hline$d, \AA$ & 1.89 & 1.99 & 2.21 \\
\hline$V_{2}, \mathrm{eV}$ & 6.87 & 6.20 & 5.02 \\
\hline \multirow{2}{*}{$V_{3}, \mathrm{eV}$} & 1.88 & 1.93 & 2.41 \\
& 1.42 & 1.37 & 1.77 \\
\hline$\alpha_{\mathrm{c}}$ & 0.96 & 0.95 & 0.90 \\
& 0.98 & 0.98 & 0.94 \\
\hline \multirow{2}{*}{$\alpha_{\mathrm{p}}$} & 0.26 & 0.30 & 0.44 \\
& 0.20 & 0.22 & 0.33
\end{tabular}

Таблица 2. Значения линейных $\chi_{1}^{\mathrm{el}}, \chi_{1}$ и квадратичных $\chi_{14}^{\mathrm{el}}, \chi_{14}$ восприимчивостей, $\varepsilon_{\infty}, \vartheta, \varepsilon_{0}$. Верхний ряд значений - расчет по таблицам Манна [11], нижний ряд — расчет по таблицам Хермана-Скиллмана [9]

\begin{tabular}{c|c|c|c}
\hline Кристалл & $\mathrm{SiC}$ & $\mathrm{GeC}$ & $\mathrm{SnC}$ \\
\hline \multirow{2}{*}{$\chi_{1}^{\mathrm{el}}$} & 0.43 & 0.44 & 0.42 \\
& 0.46 & 0.49 & 0.48 \\
\hline \multirow{2}{*}{$\chi_{1}$} & 0.48 & 0.51 & 0.58 \\
& 0.49 & 0.53 & 0.57 \\
\hline \multirow{2}{*}{$\varepsilon_{\infty}$} & 6.46 & 6.57 & 6.28 \\
& 6.81 & 7.11 & 7.00 \\
\hline \multirow{2}{*}{$\vartheta$} & 0.11 & 0.15 & 0.39 \\
& 0.06 & 0.08 & 0.19 \\
\hline \multirow{2}{*}{$\varepsilon_{0}$} & 7.00 & 7.39 & 8.29 \\
& 7.13 & 7.63 & 8.18 \\
\hline \multirow{2}{*}{$\chi_{14}^{\mathrm{el}}$} & 1.86 & 2.54 & 4.59 \\
& 1.60 & 2.12 & 4.08 \\
\hline \multirow{2}{*}{$\chi_{14}$} & 0.14 & 0.25 & 1.10 \\
& 0.07 & 0.10 & 0.50
\end{tabular}

элементарный заряд, $\gamma$ - масштабный множитель, учитывающий поправки на локальное поле и используемый как подгоночный параметр $[9,12,13]$. Для высокочастотной $\varepsilon_{\infty}$ и статической $\varepsilon_{0}$ диэлектрических проницаемостей имеем

$$
\varepsilon_{\infty}=1+4 \pi \chi_{1}^{\mathrm{el}}, \quad \varepsilon_{0}=1+4 \pi \chi_{1} .
$$

Положив $a=4.36,4.59$ и $5.11 \AA$ соответственно для $\mathrm{SiC}, \mathrm{GeC}$ и $\mathrm{SnC}[3]$, получим значения исходных параметров модели, представленные в табл. 1. Масштабный множитель $\gamma$ можно оценить по экспериментальным данным $\varepsilon_{\infty}=6.52$ и $\varepsilon_{0}=9.72$ для $3 \mathrm{C}-\mathrm{SiC}$ [14]. Выбирая для подгонки $\varepsilon_{\infty}$, получим значение $\gamma=1.44$, которое и будем использовать для всех рассматриваемых карбидов.

\section{3. Численные оценки диэлектрических восприимчивостей}

Для дальнейшего анализа удобно переписать выражения для $\chi_{1}^{\mathrm{el}}$ и $\chi_{14}$, в виде $\chi_{1}^{\mathrm{el}} \approx 0.26\left(d \alpha_{\mathrm{c}}^{3}\right)$ и $\chi_{14} \approx 0.67\left(d^{4} \alpha_{\mathrm{c}}^{2} \alpha_{\mathrm{p}}^{3}\right) \cdot 10^{-12} \mathrm{~m} / \mathrm{V}$, где $d$ измеряется в $\AA$. Результаты расчета представлены в табл. 2. Из табл. 2 следует, что значения $\chi_{1}^{\mathrm{el}}, \chi_{1}$ и $\varepsilon_{\infty}$ для различных $X \mathrm{C}$ близки. Причина такой близости состоит в том, что уменьшение ковалентности $\alpha_{\mathrm{c}}$ в ряду $\mathrm{SiC} \rightarrow \mathrm{SnC}$ компенсируется ростом $d$. Увеличение $\varepsilon_{0}$ в том же ряду связано с ростом множителя $\vartheta$. Отметим, что полученные нами значения $\varepsilon_{0}$ являются, по-видимому, заниженными. Во всяком случае, так обстоит дело для $3 \mathrm{C}-\mathrm{SiC}$.

Малость квадратичных восприимчивостей $\chi_{14}^{\mathrm{el}}$ и $\chi_{14}$ соединений $X_{\mathrm{C}}$ по сравнению с полупроводниками $\mathrm{A}_{3} \mathrm{~B}_{5}$ и $\mathrm{A}_{2} \mathrm{~B}_{6}$ (см. например, табл. 5.1 в [9]) объясняется низкой полярностью связей $\alpha_{\mathrm{p}}$. Рост $\chi_{14}^{\mathrm{el}}$ и $\chi_{14}$ при переходе от $\mathrm{SiC}$ к $\mathrm{SnC}$ связан с увеличением как $\alpha_{\mathrm{p}}$, так и $d$. Наибольшие значения $\chi_{14}^{\mathrm{el}}$ и $\chi_{14}$ соответствуют $3 \mathrm{C}-\mathrm{SnC}$. Легко показать, что максимальное значение $\chi_{14}^{\mathrm{el}}$ имеет место при $\alpha_{\mathrm{c}}^{*}=\sqrt{4 / 5}$ и $\alpha_{\mathrm{p}}^{*}=\sqrt{1 / 5}$, практически совпадающих с ковалентностью и полярностью связи $\mathrm{Sn}-\mathrm{C}$.

Необходимо отметить, что в настоящей работе мы игнорировали металличность межатомных связей $[9,10]$, учет которой, вообще говоря, может заметно сказаться на результатах расчета $[11,12]$.

\section{4. Оптические свойства}

Линейные электрооптические коэффициенты $r_{41}$ и $r_{41}^{\mathrm{el}}$, описывающие соответственно изменение показателя преломления $n=\sqrt{\varepsilon_{\infty}}$ нецентросимметричных кристаллов в низкочастотном электрическом поле и электронный вклад в $r_{41}$, определяются, согласно [11,12], как

$$
r_{41}=-4 \pi \chi_{14} / n^{4}, \quad r_{41}^{\mathrm{el}}=-4 \pi \chi_{41}^{\mathrm{el}} / n^{4}
$$

Результаты расчета представлены в табл. 3. Легко понять, что характер изменения коэффициентов $r_{41}^{\mathrm{el}}$ и $r_{41}$ в ряду карбидов определяется квадратичными восприимчивостями $\chi_{14}^{\mathrm{el}}$ и $\chi_{14}$. Полученные нами значения $\left|r_{41}^{\mathrm{el}}\right|$ и $\left|r_{41}\right|$ малы по сравнению с другими материалами (см., например, табл. 77.2 в [15]), что связано с малой полярностью связей $X-\mathrm{C}$.

Согласно [16], фотоупругие постоянные $p_{i j}(i=1,4$; $j=1,2,4)$ кубических тетраэдрических кристаллов имеют вид

$$
\begin{gathered}
p_{11}=\xi\left(1+\frac{8 \lambda}{8+\lambda}\right), \quad p_{12}=\xi\left(1-\frac{4 \lambda}{8+\lambda}\right), \\
p_{44}=\frac{99 \xi \lambda}{(8+\lambda)(8+3 \lambda)},
\end{gathered}
$$

где $\xi=-2 \eta\left(\varepsilon_{\infty}-1\right) / 3 \varepsilon_{\infty}^{2}, \eta=2\left(1-3 \alpha_{\mathrm{p}}^{2}\right), \lambda=0.85$. Результаты расчета $p_{i j}$, приведенные в табл. 3, близ- 
ки к фотоупругим постоянным алмаза $p_{11}=-0.31$, $p_{12}=-0.09$ и $p_{44}=-0.12$ (см. табл. 77.1 в [15]). Незначительный спад значений $\left|p_{i j}\right|$ в рядах $\mathrm{SiC} \rightarrow \mathrm{SnC}$ объясняется ростом полярности связей.

\section{5. Зависимость диэлектрических проницаемостей $\varepsilon_{\infty}$ и $\varepsilon_{0}$ от давления $P$}

В работе [17] показано, что

$$
\begin{gathered}
\frac{\partial \varepsilon_{\infty}}{\partial P}=-\eta \frac{\varepsilon_{\infty}-1}{3 B}, \frac{\partial \varepsilon_{0}}{\partial P}=\left(\varepsilon_{\infty}-1\right) \frac{\partial \vartheta}{\partial P}+(1+\vartheta) \frac{\partial \varepsilon_{\infty}}{\partial P}, \\
\frac{\partial \vartheta}{\partial P}=-\frac{2 \alpha_{\mathrm{p}}^{2}}{\alpha_{\mathrm{c}}^{2} B}\left(1-\frac{2 \alpha_{\mathrm{p}}^{2}}{3 \alpha_{\mathrm{c}}^{2}}\right),
\end{gathered}
$$

где $B$ - объемный модуль сжатия. В модели связывающих орбиталей Харрисона $B=2 \alpha_{\mathrm{c}}^{3} V_{2} / \sqrt{3} d^{3}$ [9]. Результаты расчета представлены в табл. 3. Следует подчеркнуть, что приведенные в табл. 3 значения $\left|\partial \varepsilon_{\infty} / \partial P\right|$ и $\left|\partial \varepsilon_{0} / \partial P\right|$ являются оценками по максимуму, так как использованный вариант расчета (без учета короткодействующего отталкивания [18]) занижает величину

Таблица 3. Значения линейных электрооптических коэффициентов $r_{14}^{\mathrm{el}}, r_{41}$, упругооптических постоянных $p_{i j} i=1,4$; $j=1,2,4$, объемных модулей сжатия $B$ и производных диэлектрических проницаемостей по давлению $\partial \varepsilon_{\infty} / \partial P$ и $\partial \varepsilon_{0} / \partial P$. Верхний ряд значений — расчет по таблицам Манна [11],

\begin{tabular}{|c|c|c|c|}
\hline Кристалл & $\mathrm{SiC}$ & $\mathrm{GeC}$ & $\mathrm{SnC}$ \\
\hline$-r_{14}^{\mathrm{el}}, 10^{-12} \mathrm{~m} / \mathrm{V}$ & $\begin{array}{l}0.55 \\
0.40\end{array}$ & $\begin{array}{l}0.74 \\
0.53\end{array}$ & $\begin{array}{l}1.46 \\
1.05\end{array}$ \\
\hline$-r_{41}, 10^{-12} \mathrm{~m} / \mathrm{V}$ & $\begin{array}{l}0.04 \\
0.02\end{array}$ & $\begin{array}{l}0.07 \\
0.02\end{array}$ & $\begin{array}{l}0.35 \\
0.13\end{array}$ \\
\hline$-p_{11}$ & $\begin{array}{l}0.25 \\
0.26\end{array}$ & $\begin{array}{l}0.22 \\
0.24\end{array}$ & $\begin{array}{l}0.14 \\
0.19\end{array}$ \\
\hline$-p_{12}$ & $\begin{array}{l}0.08 \\
0.08\end{array}$ & $\begin{array}{l}0.07 \\
0.08\end{array}$ & $\begin{array}{l}0.04 \\
0.06\end{array}$ \\
\hline$-p_{44}$ & $\begin{array}{l}0.13 \\
0.13\end{array}$ & $\begin{array}{l}0.11 \\
0.12\end{array}$ & $\begin{array}{l}0.07 \\
0.10\end{array}$ \\
\hline$B, \mathrm{GPa}$ & $\begin{array}{l}166 \\
177\end{array}$ & $\begin{array}{l}124 \\
137\end{array}$ & $\begin{array}{l}64 \\
72\end{array}$ \\
\hline$-\partial \varepsilon_{\infty} / \partial P, 10^{-2} \mathrm{GPa}^{-1}$ & $\begin{array}{l}1.74 \\
1.93\end{array}$ & $\begin{array}{l}2.19 \\
2.55\end{array}$ & $\begin{array}{l}2.13 \\
3.75\end{array}$ \\
\hline$-\partial \varepsilon_{0} / \partial P, 10^{-2} \mathrm{GPa}^{-1}$ & $\begin{array}{l}2.43 \\
2.49\end{array}$ & $\begin{array}{l}3.46 \\
3.19\end{array}$ & $\begin{array}{l}7.13 \\
6.71\end{array}$ \\
\hline$-\left(\partial \varepsilon_{\infty} / \partial P\right) / B$ & $\begin{array}{l}2.89 \\
3.41\end{array}$ & $\begin{array}{l}2.71 \\
3.48\end{array}$ & $\begin{array}{l}1.36 \\
2.70\end{array}$ \\
\hline$-\left(\partial \varepsilon_{0} / \partial P\right) / B$ & $\begin{array}{l}4.03 \\
4.41\end{array}$ & $\begin{array}{l}4.29 \\
4.37\end{array}$ & $\begin{array}{l}4.56 \\
4.83\end{array}$ \\
\hline
\end{tabular}
нижний ряд - расчет по таблицам Хермана-Скиллмана [9] объемного модуля сжатия. Действительно, для 3C-SiC по данным табл. 4.6 из [19] имеем $B=246 \mathrm{GPa}$, что в $\sim 1.5$ раза превышает полученный нами результат. Поэтому мы добавили в табл. 3 результаты расчета безразмерных производных $\left(\partial \varepsilon_{\infty} / \partial P\right) B$ и $\left(\partial \varepsilon_{0} / \partial P\right) B$, позволяющие по известным (из эксперимента или расчетов из первых принципов) значениям объемного модуля сжатия определить величины $\partial \varepsilon_{\infty} / \partial P$ и $\partial \varepsilon_{0} / \partial P$.

В заключение данного раздела отметим, что в рамках модели Харрисона имеет место соотношение

$$
\frac{\varepsilon_{\infty}-1}{\varepsilon_{0}+1}=1+\vartheta
$$

Воспользовавшись формулой Лидейна-Сакса-Теллера $\omega_{\mathrm{TO}}^{2}(0) / \omega_{\mathrm{LO}}^{2}(0)=\varepsilon_{\infty} / \varepsilon_{0}[19]$, где $\omega_{\mathrm{TO}}^{2}(0)$ и $\omega_{\mathrm{LO}}^{2}(0)-$ частоты поперечных и продольных оптических фононов в центре зоны Бриллюэна, получим [16]:

$$
\begin{gathered}
\frac{\partial \omega_{\mathrm{TO}}(0)}{\partial P}=\frac{\omega_{\mathrm{TO}}(0)}{3 B}\left(2+3 \alpha_{\mathrm{p}}^{2}\right), \\
\frac{\partial \omega_{\mathrm{LO}}(0)}{\partial P}=\frac{\omega_{\mathrm{LO}}(0)}{2 \sqrt{\varepsilon_{\infty} \varepsilon_{\infty}}}\left(\frac{\partial \varepsilon_{0}}{\partial P}-\frac{\varepsilon_{0}}{\varepsilon_{\infty}} \frac{\partial \varepsilon_{\infty}}{\partial P}\right) \\
+\sqrt{\frac{\varepsilon_{0}}{\varepsilon_{\infty}}} \frac{\partial \omega_{\mathrm{TO}}(0)}{\partial P} .
\end{gathered}
$$

Таким образом, используя соотношения (7) и (9), можно по зависимостям диэлектрических проницаемостей (оптических частот) от давления определять соответствующие зависимости для оптических частот (диэлектрических проницаемостей).

\section{6. Заключение}

В настоящей работе в рамках единого подхода получены значения целого ряда диэлектрических и оптических характеристик кубических карбидов IV группы. Насколько известно авторам, для $\mathrm{GeC}$ и $\mathrm{SnC}$ такие оценки до сих пор отсутствовали. Простота модели связывающих орбиталей Харрисона позволила нам получить для этих характеристик аналитические выражения. При этом параметры модели $d$ и $\varepsilon_{\mathrm{s}}(p)$, представляющей собой упрощенный вариант метода ЛКАО, не являются подгоночными параметрами. Отметим, что не только кристаллы 3C-SiC представляют прикладной интерес. Так, на базе этих материалов создаются сверхрешетки, например, $\mathrm{GeC} / \mathrm{SiC}, \mathrm{SnC} / \mathrm{SiC}, \mathrm{SnC} / \mathrm{GeC}$ [21] и $\mathrm{GeC} / \mathrm{GaN}$ [22].

\section{Финансирование работы}

А.А. Лебедев благодарен РФФИ за финансовую поддержку (грант РФФИ № 20-0200117).

\section{Конфликт интересов}

Авторы заявляют, что у них нет конфликта интересов. 


\section{Список литературы}

[1] А.А. Лебедев, П.А. Иванов, М.Е. Левинштейн, Е.Н. Мохов, С.С. Нагалюк, А.Н. Анисимов, П.Г. Баранов. УФН 189, 8, 803 (2019).

[2] M.E. Levinshtein, S.L. Rumyantsev, M.S. Shur. Properties of Advanced Semiconductor Materials: GaN, AIN, InN, BN, SiC, SiGe. Wiley, N. Y. (2001).

[3] R. Pandey, M. Rérat, M.C. Darrigan, M. Causá. J. Appl. Phys. 88, 11, 6462 (2000).

[4] A. Benzair, H. Aourag. Phys. Status Solidi B 231, 2, 411 (2002).

[5] W. Sekkal, A. Zaoui. New J. Phys. 4, 1, 9 (2002).

[6] A. Mahmood, L.E. Sansores. J. Mater. Res. 20, 5, 1101 (2005).

[7] A. Hao, X. Yang, X. Wang, Y. Zhu, X. Liu, R. Liu. J. Appl. Phys. 108, 6, 063531 (2010).

[8] R. Muthaiah, J. Garg. arXiv: 2107.04596

[9] У. Харрисон. Электронная структура и свойства твердыХ тел. Мир, М. (1983). Т. 1.

[10] W.A. Harrison. Phys. Rev. B 27, 6, 3592 (1983).

[11] W.A. Harrison. Phys. Rev. B 31, 4, 2121 (1985).

[12] С.Ю. Давыдов, Е.И. Леонов. ФТТ 30, 5, 1326 (1988).

[13] С.Ю. Давыдов, С.К. Тихонов. ФТТ 37, 10, 3044 (1995).

[14] В.И. Гавриленко, А.М. Грехов, Д.В. Корбутяк, В.Г. Литовченко. Оптические свойства полупроводников. Справочник. Наук. думка, Киев (1987).

[15] Ю.И. Сиротин, М.П. Шаскольская. Основы кристаллофизики. Наука, М. (1975).

[16] С.Ю. Давыдов, С.К. Тихонов. ФТП 31, 7, 823 (1997).

[17] С.Ю. Давыдов, С.К. Тихонов. ФТП 32, 9, 1057 (1998).

[18] F. Bechstedt, W.A. Harrison. Phys. Rev. B 39, 8, 5041 (1989).

[19] С.П. Никаноров, Б.К. Кардашов. Упругость и дислокационная неупругость кристаллов. Наука, М. (1985).

[20] Ч. Киттель. Введение в физику твердого тела. Наука, М. (1978).

[21] Ю.М. Басалаев, Е.Н. Малышева. ФТП 51, 647 (2017).

[22] P. Lou, J.Y. Lee. ACS Appl. Mater. Interfaces 12, 12, 14289 (2020).

Редактор Е.В. Толстякова 